\title{
On heat transfer variation in film flow related with surface cross curvature
}

\author{
S. Sinkunas ${ }^{1}$, J. Gylys $^{1} \&$ A. Kiela ${ }^{2}$ \\ ${ }^{1}$ Department of Thermal and Nuclear Energy, \\ Kaunas University of Technology, Lithuania \\ ${ }^{2}$ Department of Technology, Kaunas College, Lithuania
}

\begin{abstract}
This paper is concerned with heat transfer calculation in a liquid film falling down a vertical surface. Heat transfer in laminar film flow is influenced by the cross curvature of the wetted surface, heat flux on the film surface and boundary conditions on the wetted wall. The analytical study evaluating the influence of cross curvature on heat transfer in laminar film has been carried out. Equations using correction factors for the calculation of heat transfer in laminar liquid film with respect to the cross curvature for different boundary conditions were established. An experimental investigation of heat transfer in the entrance region of the turbulent film has also been performed. The description of the experimental set-up is presented in the paper. The research has been carried out on a water film flowing down a surface of vertical tube with the Reynolds number ranging from $9.2 \cdot 10^{3}$ to $10.5 \cdot 10^{3}$. The results of experiments are discussed with respect to the local heat transfer dependence on the Reynolds number and initial velocity of the film. Heat transfer stabilization length was determined experimentally.

Keywords: heat transfer, laminar film, cross curvature, correction factor, turbulent film, entrance region.
\end{abstract}

\section{Introduction}

In many technological processes that deal with heat and mass transfer, gravity driven liquid films are widely used. With liquid falling films one can obtain comparatively high heat transfer coefficient. This approach is employed for cooling and heating processes in chemical, food, pharmaceutical and other 
industries. Prediction of heat transfer in liquid film flow is important from various engineering aspects. Even small improvements in heat transfer during technological processes may lead to significant energy and money savings. The growing awareness of environmental problems recently draw the efforts of many researches, including the field of engineering, to evaluate systems on the basis of heat transfer phenomena.

A lot of the heat exchange equipments are designed of vertical tubes with falling films on their external surfaces. In the case when liquid film flows down a vertical tube the curvature of its surface and the film itself effects heat transfer characteristics on the surface and correspondingly thickness of the liquid film [1]. Simultaneously the intensity of the heat exchange between a wetted surface and liquid film is influenced.

The heat transfer mechanism of a liquid film flowing down profiled horizontal tubes was studied in several works [2-3]. A method of its enhancement based on the breakdown of the thermal boundary layer by longitudinal fins and grooves was developed. The results of heat transfer research in evaporating falling films on a structured and smooth surface respectively are presented in [4-5]. The obvious change of the wavy film structure by using a profiled heating surface showed the improvement of heat transfer coefficient. Hydrodynamic and thermal liquid film flow study on vertical plate with grids was performed in [6]. The conditions for an optimum heat transfer in evaporating water film driven by gravity down a vertical heated plate were established. It was evaluated the influence of wetted surface geometry and operating parameters on the heat transfer enhancement.

\section{Analytical method}

\subsection{Heat transfer in laminar film flow on a vertical surface}

Heat transfer across the liquid film takes place due to the temperature difference between the wetted surface and the film. In the case of laminar film flow the conduction may be viewed as a main mode of the heat transfer only. Thus, the equation known as Fourier's law can be used to compute the amount of energy being transferred per unit time

$$
q=-\lambda \operatorname{grad} T
$$

By substituting the expression of the temperature gradient for eqn. (1) and integrating, we obtain

$$
T_{w}-T=\frac{q_{w}}{\lambda} \int_{0}^{y} \frac{q}{q_{w}} d y .
$$

The heat flux from a liquid film can be determined as follows 


$$
Q=c T_{f} \rho \bar{w} b \delta=\int_{0}^{\delta} c T \rho w b d y
$$

The mean temperature can be defined as

$$
T_{f}=\frac{\int_{0}^{\delta} w T d y}{\bar{w} \delta} .
$$

By defining the temperature difference between a wetted surface and the mean temperature of the film as

$$
T_{w}-T_{f}=\frac{\frac{q_{w}}{\lambda} \int_{0}^{\delta} w\left(\int_{0}^{y} \frac{q}{q_{w}} d y\right) d y}{\bar{w} \delta},
$$

we obtain the following expression for calculation of local heat transfer coefficient

$$
\alpha=\frac{\bar{w} \delta \lambda}{\int_{0}^{\delta} w\left(\int_{0}^{y} \frac{q}{q_{w}} d y\right) d y}
$$

In order to define the regularity $q / q_{w}=f(y)$, we use the energy equation

$$
c \rho w \frac{\partial T}{\partial x}+\frac{d q}{d y}=0 .
$$

By integrating the eqn. (7) within the limits from 0 to $y$, we obtain the ratio of heat flux densities in the film

$$
\frac{q}{q_{w}}=1-\frac{c \rho}{q_{w}} \int_{0}^{y} w \frac{\partial T}{\partial x} d y .
$$

Assume that $\partial T / \partial x=d T_{f} / d x$ [7]. Then the ratio $d T_{f} / d x$ can be determined by the equation of heat balance in the film 
234 Advanced Computational Methods in Heat Transfer IX

$$
\frac{d T_{f}}{d x}=\frac{q_{w}}{c \rho \bar{w} \delta} .
$$

Substituting of eqn. (9) by eqn. (8) leads to the following relationship

$$
\frac{q}{q_{w}}=1-\frac{\int_{0}^{y} w d y}{\bar{w} \delta} .
$$

By substituting eqn. (10) for eqn. (6) and by employing the Nusselt number $N u_{d}$, we obtain

$$
N u=\frac{\bar{w} \delta d}{\int_{0}^{\delta} w\left(\int_{0}^{y}\left(1-\frac{\int_{0}^{y} w d y}{\bar{w} \delta}\right) d y\right) d y} .
$$

Velocity in the laminar film flow one can calculate by the following equation

$$
w=\frac{g \delta y}{v}\left(1-0.5 \frac{y}{\delta}\right),
$$

and the mean velocity respectively

$$
\bar{w}=\frac{g \delta^{2}}{3 v} .
$$

Taking into account eqns. (12) and (13), we obtain the following expression from eqn. (11)

$$
N u_{d}=\frac{8 w_{s} \delta^{2}}{3 \int_{0}^{\delta} w\left(\int_{0}^{y}\left(1-\frac{3 \int_{0}^{y} w d y}{2 w_{s}}\right) d y\right) d y}
$$


The above-mentioned equation could be solved numerically and the following result is obtained

$$
N u_{d}=8.2353
$$

In case of heat transfer calculations, it is more reasonable to use modified Nusselt number $N u_{M}$. Therefore, the following equation can be used

$$
N u_{M}=2.27 R e^{-1 / 3}
$$

As it is shown in [8], the application of eqn. (16) can be expanded using the corresponding multipliers. The curvature of the film flowing down a vertical tube surface and the heat transfer between film surface and surrounding medium of a gas or vapour, can be evaluated by correction factor $C_{R q}$. Multiplier $\varepsilon_{P r}$ may estimate the variability of liquid physical properties. Then, the modified Nusselt number can be calculated by the following equation

$$
N u_{M}=2.27 R e^{-1 / 3} C_{R q} \varepsilon_{P r} .
$$

The correction factor $C_{R q}$ can be determined by equations: when $q_{w}=$ const

$$
C_{R q}=\frac{136}{136+39 \varepsilon_{q}}+\left(0.52-0.03 \varepsilon_{q}\right) \varepsilon_{R}
$$

and when $T_{w}=$ const

$$
C_{R q}=\frac{56}{56+10 \varepsilon_{q}}+\left(0.58-0.05 \varepsilon_{q}\right) \varepsilon_{R} .
$$

The variations of correction factor $C_{R q}$ upon the range of relative cross curvature and external heat exchange between the film surface and surrounding medium according to eqns. (18) and (19) are shown in figure 1.

The variation of liquid physical properties across the film must be taken into account in a case of high value of temperature difference. The multiplier $\varepsilon_{P r}$ in eqn. (17) can be determined as an exponential function of the following ratios $\operatorname{Pr}_{f} / \operatorname{Pr}{ }_{w}$ or $\mu_{f} / \mu_{w}$. The results of numerical calculations showed that ratio $\operatorname{Pr}_{f} / \operatorname{Pr}_{w}$ unambiguously does not evaluates the influence of physical properties variation for different liquids and the character of this influence practically is not dependent upon the values of $\varepsilon_{q}$ and $\varepsilon_{R}$. This research showed that for $-0.5 \leq \varepsilon_{R} \leq-1$ and $0.4 \leq \operatorname{Pr}_{f} / \operatorname{Pr}_{w} \leq 2$ the multiplier $\left(\operatorname{Pr}_{f} / \operatorname{Pr}_{w}\right)^{0.25}$ can be 
used. The multiplier $\varepsilon_{P r}$ must be determined as a function of parameters $\mu_{f} / \mu_{w}$ for more accurate evaluation of liquid physical properties variation. This parameter evaluates practically the influence of physical properties variation for water, transformer oil, fuel oil and compressor oil. The multiplier $\varepsilon_{P r}$ in such case can be calculated by equation

$$
\varepsilon_{P r}=\left(\mu_{f} / \mu_{w}\right)^{n},
$$

where $n=0.315\left(2+\varepsilon_{R}\right)^{-0.49}$, for $0.1 \leq \mu_{f} / \mu_{w} \leq 1$ and $n=0.325\left(2+\varepsilon_{R}\right)^{-0.24}$, for $1 \leq \mu_{f} / \mu_{w} \leq 10$. The similar data in [8] for $\varepsilon_{R}=0$ have showed that boundary conditions on the wetted surface have no influence to the character of heat transfer dependence on variability of the liquid physical properties.
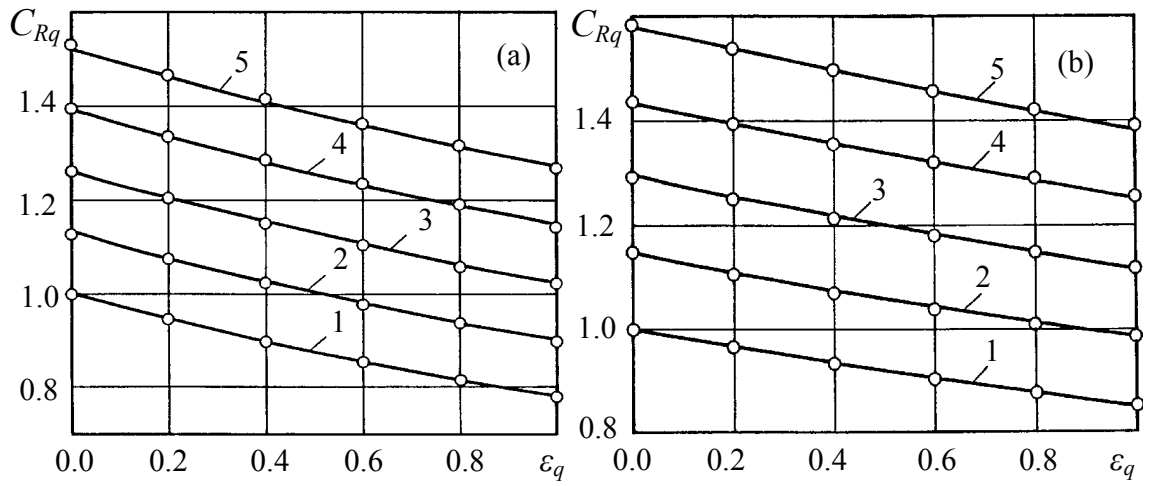

Figure 1: Variations of correction factor on film cross curvature and external heat transfer: (a) boundary condition $q_{w}=$ const and (b) boundary condition $T_{w}=$ const $1-5-\varepsilon_{R}=0 ; 0.25 ; 0.50 ; 0.75$ and 1.0 correspondingly.

\section{Experimental method}

\subsection{Experimental set-up}

For heat transfer research in the turbulent film falling down a surface of vertical tube the experimental set-up (figure 2) was applied.

The arrangement was composed of a closed circulating loop that included liquid reservoir with feed and exhaust electric pumps and liquid tank. Working liquid with the aid of feed pump was supplied to the liquid tank provided with a slot distributive mechanism. The liquid from the distributor fell down a vertical tube (calorimeter). The stainless steel tube $30 \mathrm{~mm}$ in outside diameter with the length of $1000 \mathrm{~mm}$ was used in the experiment as a calorimeter. The fixing bolts 
at the end of tested tube allowed the possibility to regulate and to guarantee verticality of the tube. After flowing down the test tube, water was gathered back to the reservoir. The gutter at calorimeter end ensured a smooth falling of the water into the reservoir. The surplus water was discharged to the sewerage by exhaust pump while the fresh water was supplied from water-supply directly. The location of thermocouple in the liquid distributor ensured the measurement of film temperature at the inlet. The thermocouple installed at the end of calorimeter had determined a film temperature at the exit correspondingly. The electric circuit consisted of calorimeter, voltage regulator, and shunt with milivoltmeter, rectifier and voltmeter respectively. The electric current supplied for the calorimeter provided a steady heat flux on the experimental section.

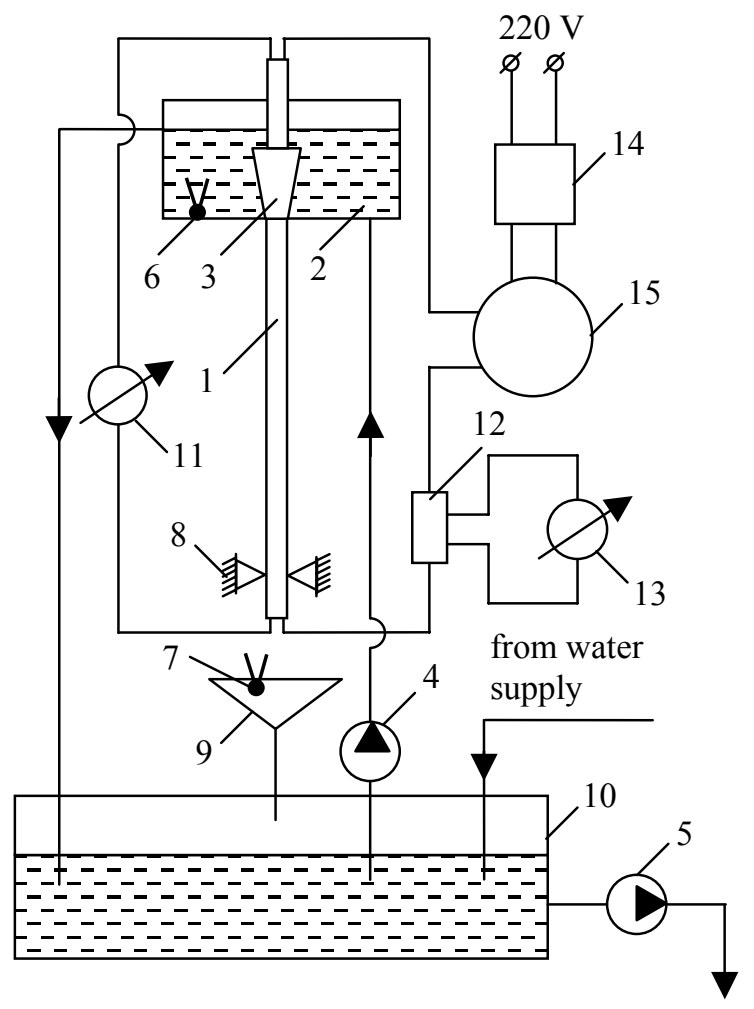

Figure 2: Schematic diagram of experimental set-up: 1 - calorimeter; 2 - liquid tank; 3 - slot distributive mechanism; 4 - feed-pump; 5 - exhaust-pump; 6 - inlet thermocouple; 7 - outlet thermocouple; 8 - centering bolts; 9 - gutter; 10 - liquid reservoir; 11 - voltmeter; 12 - shunt; 13 - millivoltmeter; 14 - voltage regulator; 15 - rectifier. 


\subsection{Local heat transfer for a turbulent film flow on a vertical surface}

The experiments were provided for Reynolds number ranged from $9.2 \cdot 10^{3}$ to $10.5 \cdot 10^{3}$. The temperature of the tube (calorimeter) surface and the film, electric current, voltage were measured and recorded during the experiment. After registration of electric current $I$ and voltage $U$, the heat flux density on the calorimeter surface was calculated dividing of heating power $I \times U$ by surface area $A$. When records of heated tube surface and film flow temperatures were performed, the difference of temperature $\Delta T$ (between the mean temperatures of film $\bar{T}_{f}$ and tube surface $T_{w}$ ) was calculated. Local heat transfer coefficient was computed by formula

$$
\alpha=q_{w} / \Delta T
$$

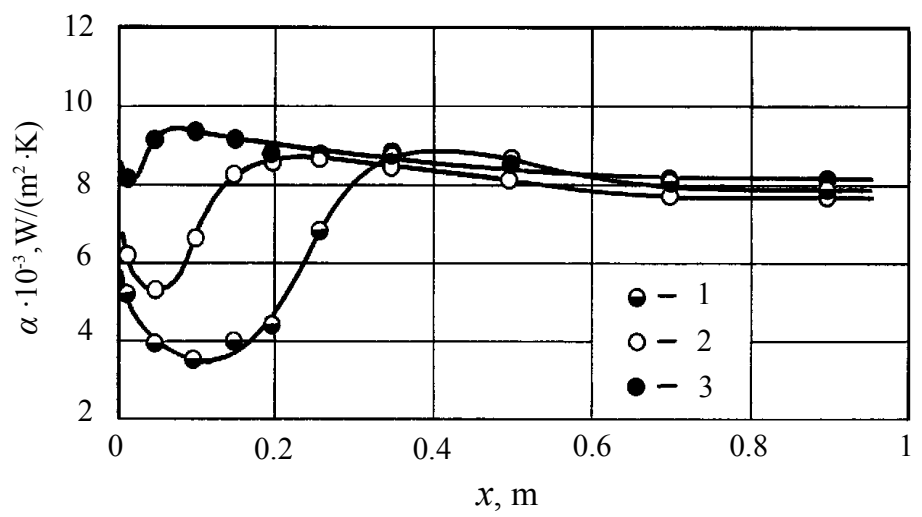

Figure 3: Variation of local heat transfer coefficient in the entrance region of the film flow down a vertical surface: $1-\mathrm{Re}=9240, \varepsilon=0.89$; $2-\operatorname{Re}=9300, \varepsilon=1.15 ; 3-\operatorname{Re}=10540, \varepsilon=1.2$.

Experimental data are presented in figure 3. Three different regions may be distinguished along the length of film flow in a case when initial average velocity of the film in the liquid distributor is less or exceeds an average velocity of stabilized flow. The significant decrease of local heat transfer coefficient while reaching minimal value at some distance from liquid distributor is seen at the first region. This phenomenon one can explain by the development of thermal boundary layer and its laminar nature. In the second region, fluid fluctuations begin to develop while heat transfer increases to a maximum value. The beginning of heat transfer stabilization takes place in the third region of the film flow. Augmentation of a thermal boundary layer terminates with the film thickness. The variation of local heat transfer in the entrance region, when initial average velocity of the film exceeds an average velocity of stabilized film is not so high. As we can see from figure 3, in all cases the heat transfer stabilization is 
not larger than $0.5 \mathrm{~m}$ from the liquid distributor when Reynolds number ranged from $9.2 \cdot 10^{3}$ to $10.5 \cdot 10^{3}$.

\section{Conclusions}

The cross curvature and external heat exchange of the film has a significant influence on heat transfer rate from the wetted surface in laminar gravitational liquid film flowing down the outside surface of vertical tube. In this case equation for heat transfer intensity calculation in laminar liquid film falling down a vertical plane surface must be supplemented with multiplier $C_{R q}$ evaluating curvature of film and with multiplier $\varepsilon_{P r}$ evaluating the variability of liquid physical properties respectively.

The turbulent flow can be useful in the sense of providing increased heat transfer intensity. However, the turbulent film flow is very complicated and difficult for the theoretical study. In this case, the only possible way is the experiment. The experimental data revealed that Reynolds number and initial velocity has a significant influence on local heat transfer. It is estimated that heat transfer stabilization takes place at $0.5 \mathrm{~m}$ distance from the liquid distributor when $\operatorname{Re}>9 \cdot 10^{3}$.

\section{Nomenclature}

$a$ - thermal diffusivity, $\mathrm{m} / \mathrm{s}^{2} ; b$ - elementary width of the film; $c$ - specific heat, $\mathrm{J} /(\mathrm{kg} \cdot \mathrm{K}) ; C_{R q}$ - heat transfer correction factor; $d$ - hydraulic diameter of the film, $\mathrm{m} ; g$ - acceleration of gravity, $\mathrm{m} / \mathrm{s}^{2} ; N u$ - Nusselt number, $\alpha \delta / \lambda ; N u_{d}$ - Nusselt number, $\alpha d / \lambda ; N u_{M}$ - modified Nusselt number, $(\alpha / \lambda)\left(v^{2} / g\right) ; \operatorname{Pr}-$ Prandtl number, $v / a ; q$ - heat flux density, $\mathrm{W} / \mathrm{m}^{2}$; Re - Reynolds number of liquid film, $4 \Gamma /(\rho v) ; T$ - temperature, $\mathrm{K} ; w$ - film velocity, $\mathrm{m} / \mathrm{s} ; x$ - longitudinal coordinate; $y$ - distance from the wetted surface, $\mathrm{m} ; \alpha$ - heat transfer coefficient, $\mathrm{W} /\left(\mathrm{m}^{2} \cdot \mathrm{K}\right) ; \Gamma$ - wetting density, $\mathrm{kg} /(\mathrm{m} \cdot \mathrm{s}) ; \delta$ - liquid film thickness, $\mathrm{m} ; \varepsilon$ - relative film velocity, $\bar{w}_{d} / \bar{w}_{\text {stab }} ; \varepsilon_{R}$ - relative cross curvature of the film, $\delta / R ; \varepsilon_{\mathrm{Pr}}-$ multiplier for liquid physical properties; $\varepsilon_{q}$ - ratio of heat flux densities, $\left(q_{s} / q_{w}\right) ; \mu$ - dynamic viscosity, $(\mathrm{Pa} \cdot \mathrm{s}) ; \lambda-$ thermal conductivity, $\mathrm{W} /(\mathrm{m} \cdot \mathrm{K}) ; v$ - kinematic viscosity, $\mathrm{m}^{2} / \mathrm{s} ; \rho$ - liquid density, $\mathrm{kg} / \mathrm{m}^{3}$;

Subscripts: $d$ - distributor; $f$ - film flow; $s$ - film surface; stab - stabilized flow; $w$ - wetted surface.

\section{References}

[1] Sinkunas, S., Kiela, A., Adomavicius, A. \& Gudzinskas, J., Hydromechanical parameters of laminar liquid film falling down a vertical surface. Mechanics, 4(36), pp. 24-29, 2003. 
[2] Rifert, V., Sidorenko, V., Usenko, V. \& Zolotukhin, I., Methodic of measurements and experimental results of local heat transfer at liquid film flow on the horizontal finned tubes. Proc. of the $5^{\text {th }}$ World Conf. On Experimental Heat Transfer, Fluids Mechanics and Thermodynamics, Edizioni ETS: Pisa, Vol. 3, pp.1997-2000, 2001.

[3] Rifert, V., Putilin, J. \& Podbereznyi, V., Evaporation heat transfer in liquid films flowing down horizontal smooth and longitudinally profiled tubes. J. of Enhanced Heat Transfer, 8, pp. 91-97, 2001.

[4] Lozano Aviles, M., Maun, A.H., Iversen, V., Auracher, H. \& Wozny, G., High frequency needle probes for time-and-space characteristics measurements of falling films on smooth and enhanced surfaces. Proc. of $6^{\text {th }}$ Word Conf. On Experimental Heat Transfer, Fluids Mechanics and Thermodynamics, Matsushima, Miyagi, Vol. 1, pp. 1-5, 2005.

[5] Takase, K., Yoshida, H. \& Ose, Y., Predicted thermal-hydraulic of liquid film flow on ribbed surface. Proc. of $1^{\text {st }}$ Int. Forum On Heat Transfer, Kyoto, Vol.1, pp. 207-208, 2004.

[6] Mitrovic, J. \& Raach, H., Hydrodynamic and thermal film flow study on vertical plates with grids. Report On The Progress of Easy MED Workpage 2 For The Tip, Institute for Energy and Process Engineering University of Paderborn, pp. 1-4, 2004.

[7] Kays, W.M., Convective Heat and Mass Transfer, Energy: Moscow, 1972.

[8] Gimbutis, G., Gimbutyte, I. \& Sinkunas, S., Heat transfer in a falling liquid film with large curvature. Heat Transfer Research, Scripta Technica, 2, pp. 216-219, 1993. 